\title{
The Influence of Shoe-Heel Height on Knee Muscle Activity of Transtibial Amputees During Standing
}

\author{
Xiaohong Jia ${ }^{1}$, Jichuan Zhang ${ }^{1}$, Rencheng Wang ${ }^{1}$, Lidan Fang ${ }^{1}$, Dewen Jin ${ }^{1}$ \\ and Ming Zhang ${ }^{2}$ \\ ${ }^{1}$ Division of Intelligent and Biomechanical System, State Key Laboratory of Tribology, \\ Tsinghua University, Beijing 100084, China \\ ${ }^{2}$ Department of Health Technology and Informatics, The Hong Kong Polytechnic \\ University, Hong Kong, China \\ jiaxh@mail.tsinghua.edu.cn
}

\begin{abstract}
In order to access the effects of shoe-heel height on knee muscle activity for transtibial amputees during standing, five male subjects volunteered for the study. Three pairs of shoes with zero, $20 \mathrm{~mm}$ and $40 \mathrm{~mm}$ heel height were used during normal standing. Surface EMG of 10 muscles was recorded by the Noraxon surface EMG collection system. EMG-MAV of the medial and lateral gastrocnemius of the sound leg almost change double with increase in heel height from zero to $40 \mathrm{~mm}$, and EMG-MAV of the rectus fomris, vastus lateralis and vastus medialis of prosthetic side became larger to different extent. The finding in this paper suggested that an alignment change was necessary to accommodate the heel height change and the prostheses users should be cautious to choose shoes in daily life.
\end{abstract}

Keywords: Shoe-heel height, Surface EMG, Prosthesis, Standing.

\section{Introduction}

Artificial limb is a main way to recover the activity and appearance for amputees. The performance of the prosthesis influences the level of rehabilitation for the transtibial (TT) amputees greatly. The alignment, defined as the relative position and orientation of the prosthetic components, has been recognized as one main factor that determines the success of a prosthesis for TT amputees [1]. Misalignment always results in deviation of standing pattern and gait, increase of energy cost, pain or even tissue breakdown at the socket/stump interface.

Lots of work has been done to obtain a more quantitative technique and optimization criteria for TT prosthetic alignment [2-5], which has theoretical merit to provide quantitative information to the practitioners that is difficult to obtain through their direct subjective observation, and also to enable rehabilitation outcomes to serve as valuable evidence of treatment efficacy. However, even though the prosthesis was adjusted optimally for one amputee, misalignment would still happen when prosthesis was not used properly. However, even though the prosthesis is aligned optimally for one amputee, the alignment would be changed if the prosthesis is not used properly. It 
is well accepted that persons who use lower-limb prostheses are often restricted to the choice of a narrow range of heel heights [6]. But the change of shoes with different heel heights is necessary in human daily life which may destroy the fitted alignment setting by the prosthetist. At the same time, the static standing status is a main and basic activity of the amputee's daily life, it is important to study how much the alignment influences the static standing biomechanics. The objective of this study is to look into the effect of shoe-heel height on knee muscle activity of below-knee amputee during standing, and to provide quantitative information for a biomechanically optimal prosthetic alignment.

\section{Methods}

\subsection{Subjects and Prostheses}

Five males with unilateral TT amputation participated in this work. Table 1 outlined the subject characteristics. Each subject signed an approved human subject's consent form. From each subject, a certified prosthetist duplicated his existing PTB socket with 5-mm-thick polyurethane liner, and fitted it with new shank and SACH foot with two Otto Bock adapters 4R21 (Otto Bock Orthopadische GmbH \& Co., Duderstadt, Germany). Each component can be angularly adjusted relative to each other in the antero-posterior and medio-lateral planes. The five subjects had no other concomitant disabilities and skin complications. This experienced prosthetist also joined in this study to ensure the function of each participant's prosthesis.

Table 1. Subjects data

\begin{tabular}{llllllll}
\hline $\begin{array}{l}\text { Subject } \\
\text { number }\end{array}$ & $\begin{array}{l}\text { Age } \\
\text { (years) }\end{array}$ & $\begin{array}{l}\text { Body } \\
\text { mass }(\mathrm{Kg})\end{array}$ & $\begin{array}{l}\text { Height } \\
(\mathrm{cm})\end{array}$ & $\begin{array}{l}\text { Prosthetic } \\
\text { side }\end{array}$ & $\begin{array}{l}\text { Years since } \\
\text { amputation }\end{array}$ & Cause & $\begin{array}{l}\text { Activity } \\
\text { level }\end{array}$ \\
\hline 1 & 23 & 57 & 170 & left & 6 & trauma & high \\
2 & 58 & 70 & 168 & right & 12 & trauma & moderate \\
3 & 32 & 75 & 177 & left & 3 & infection & high \\
4 & 44 & 65 & 172 & left & 11 & trauma & moderate \\
5 & 28 & 69 & 174 & left & 2 & trauma & high \\
\hline
\end{tabular}

\subsection{Experiment and Data Collection}

Three levels of shoe-heel height were investigated in this study: shoes with zero, 20 $\mathrm{mm}$ and $40 \mathrm{~mm}$ heel height. All shoes used here were commercially available. Surface EMG signals were recorded to investigate the effect of shoe-heel height on knee muscle activity, at a sampling rate of $1500 \mathrm{~Hz}$ using pre-amplified bipolar, surface electrodes (Noraxon, Scottsdale, AZ, USA), which were placed on the cleanshaven skin overlying biceps femoris, rectuc femoris, vastus lateralis, and vastus medialis of both legs and the medial and lateral gastrocnemius of the sound leg. The 
ground electrode was placed overlying the tuberosity of the tibia. Since the shoes with $20 \mathrm{~mm}$ heel height are normal choice according to subjects' responses, the prostheses were aligned to be optimal for the subjects with this height before experiment. The alignment was kept the same when the heel height was changed. Every subject was asked to load the prosthesis to $40 \%$ of body weight as measured by the Otto Bock L.A.S.A.R. Posture alignment system for each trial. The experimental set up was shown in Fig. 1. Loading line of prosthetic side, plantar foot pressure were also simultaneously recorded to reveal the effects of heel height.
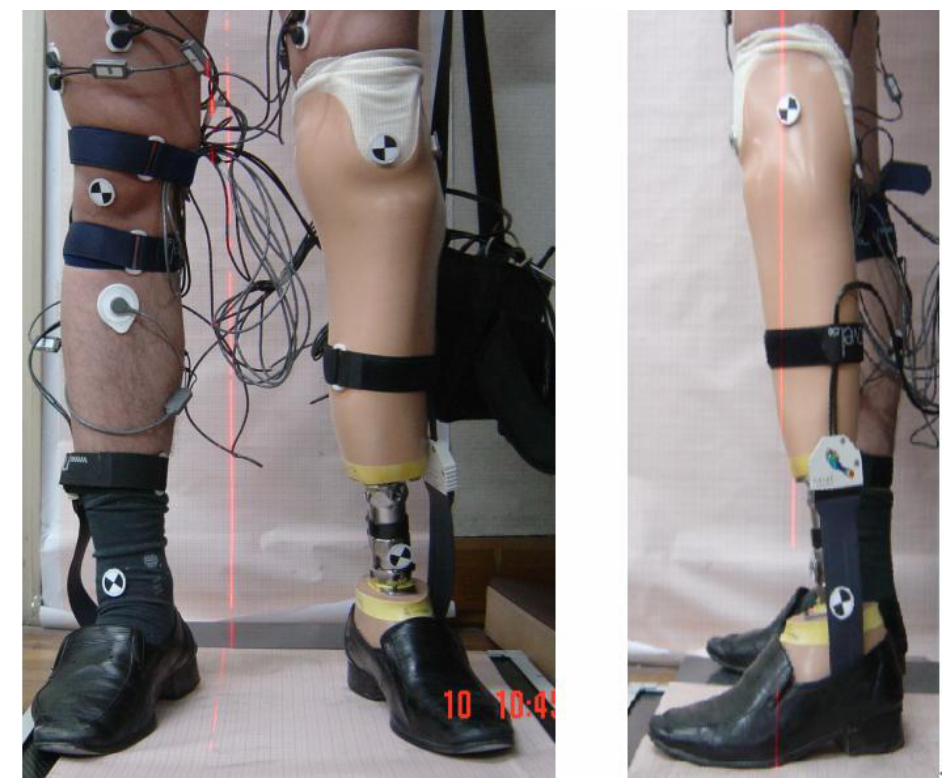

Fig. 1. Experiment system to study effect of heel height (anterior and lateral view)

The same experiment repeated twice. The shoe-heel height was changed in a random turn, and after a short break the data were re-measured. All data was recorded for 5 seconds when the subjects were asked to keep the standing posture for about 10 seconds. After each change, the subjects were asked to walk with close observation of the prosthetist to ensure the safety of the prostheses with different shoes.

\subsection{Data Analysis}

The EMG signal is widely used in analyzing the activity of muscles. Mean absolute value (MAV) of EMG was used to reveal the influence of the changes of the shoeheel height, which was defined as equation 1 . Here $x_{i}$ is signal and $\mathrm{N}$ is the number of points.

$$
\mathrm{MAV}=\frac{1}{N} \sum_{i=1}^{N}\left|x_{i}\right|
$$




\section{Results}

Fig. 2 illustrated the comparison of typical muscle activity between sound and prosthetic side (these data were from subject 1 , the others showed the same trend). In order to keep standing balance, muscles of prosthetic side were more active than the same ones of sound side. Especially for biceps femoris and vastus medialis of prosthetic side, the EMG-MAV was almost doubled comparing with sound side. This may be due to subjective adjustment. Biceps femoris and vastus medialis, which are responsible for keeping stability of knee joint, must contract more to prevent injury from hyperextension or flexion.

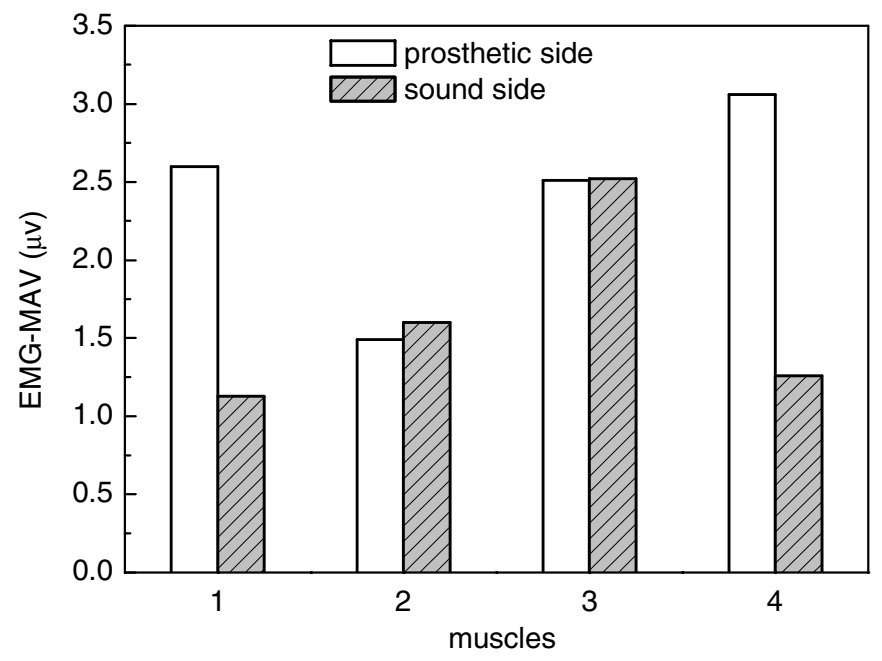

Fig. 2. The comparison of mean absolute value (MAV) of EMG of muscles of both sides, including 4 muscles (Subject 1). 1- biceps femoris, 2 -rectus femoris, 3 - vastus lateralis, 4 vastus medialis.

Fig. 3 illustrated the typical muscle activity for sound and prosthetic side related to different shoe-heel height (these data were from subject 1 too, the others showed the same trend). During static standing, the EMG signals of knee muscles of the sound leg did not change a lot when the heel height was varied, whereas EMG-MAV of the medial and lateral gastrocnemius of the same leg was almost doubled when the heelheight increased from zero to $40 \mathrm{~mm}$. This was because the two posterior caff muscles, responsible for ankle plantarflexion, played an important role in standing balance (Buckley, 2002). In contrast, the EMG of the knee muscles of the prosthetic side was systematically affected by the heel height change. When the heel height changed from zero to $40 \mathrm{~mm}$, the knee flexion angle increased as a result. In order to keep the standing balance and knee stability, the muscles for knee extension must be more active. In Fig. 3, it can be found that EMG-MAV of the rectus fomris, vastus lateralis and vastus medialis became larger with higher shoe-heel to different extents. At the same time, the EMG-MAV of the biceps femoris reduced slightly. 


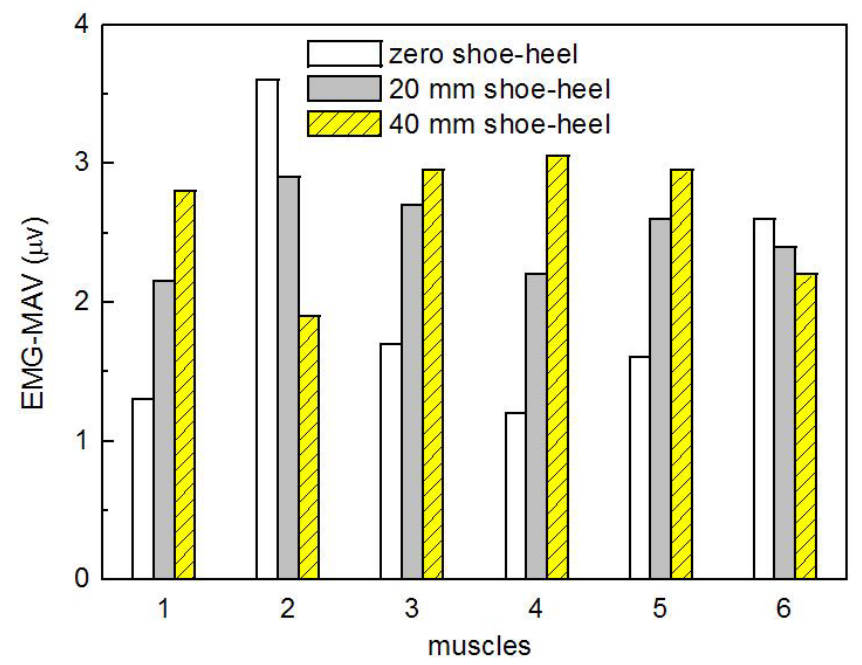

Fig. 3. Mean absolute value (MAV) of EMG of muscles change with the three heel heights, including 6 muscles (Subject 1). Sound side: 1- medial gastrocnemius, 2 - lateral gastrocnemius; Prosthetic side: 3 - rectus femoris, 4 - vastus medialis, 5 - vastus lateralis, 6 biceps femoris.

\section{Discussion}

The importance of prosthetic alignment to the success of prosthetic fitting is well known, but work remains to be done in this area. Although it was reckoned that an alignment change is necessary to accommodate higher or lower heel height when shoe-heel height changes beyond a small range [6], only the effects of shoe-heel height on the rollover characteristics of the biologic ankle-foot system for nondisabled adult females were studied. In our primary work, the effect of shoe-heel height on the knee muscle activity was studied. Since the heel-height change for the TT amputee during standing actually caused a change in orientation angle in the sagittal plane of the socket relative to the ground, our results could be explained by other reports about sagittal alignment in the literature.

When the heel height was reduced to zero, the knee joint was hyper-extended. The rectus fomris, vastus lateralis, and vastus medialis, which are responsible to extend the knee joint, provided less force when heel height was zero because the EMG amplitude was approximately proportional to muscle force when the EMG was measured under isometric conditions. On the other hand, when heel height was changed from zero to $40 \mathrm{~mm}$, an external flexion moment happened to the knee joint. To maintain the standing balance and joint stability, muscle forces of the rectus fomris, vastus lateralis, and vastus medialis had to increase. Although the posterior knee ligament relaxed, more energy consumption in the muscles could increase fatigue in the amputee. With long-term standing, some damage might occur in the knee joint. 
Standing alignment for the TT amputee has been defined as biomechanically optimal when the anatomical knee center is $15 \mathrm{~mm}$ posterior to the individual loading line of the prosthetic side [7]. In this study, the prosthesis was adjusted to optimal alignment when the heel height was $20 \mathrm{~mm}$. Under this condition, the loading line of the prosthetic side is $6 \sim 23 \mathrm{~mm}$ anterior to the knee center for five subjects, agreeing with the above optimal criterion by Blumentritt to some extent. The distance between the load line of prosthetic side and knee joint center was defined as $L$, which was positive when the load line was anterior to the knee center. It was found that $L$ was 58 $\mathrm{mm}$ for zero shoe-heel and $-32 \mathrm{~mm}$ for $40 \mathrm{~mm}$ shoe-heel (for subject 1).

In this study, the effects of shoe-heel height of transtibial amputees during standing were investigated based on knee muscle activity. Because the subjects would adjust the standing posture automatically to compensate the changes of the heel height, it may be difficult to find some directly practical criterion for prosthetic alignment. This study tried to emphasize the importance of proper use of prosthesis. The finding in this paper suggested that an alignment change was necessary to accommodate the heel height change and the prostheses users should be cautious to choose their shoes in daily life.

Acknowledgments. This work was supported by National Science Funding of China, No. 50575122. The all authors would like to thank Xiaobing Li and Yong Luo of National Rehabilitation Center of China.

\section{References}

1. Yang, L., Solomonidis, S.E., Spence, W.D.: The Influence of Limb Alignment on the Gait of Above-knee Amputees. J. Biomech. 11, 981-997 (1991)

2. Andres, R.O., Stimmel, S.K.: Prosthetic Alignment Effects on Gait Symmetry: a Case Study. Clin. Biomech. 5, 88-96 (1990)

3. Winter, D.A., Sienko, S.E.: Biomechanics of Below-knee Amputee Gait. J. Biomech. 21, 361-367 (1988)

4. Geil, M.D., Lay, A.: Plantar Foot Pressure Responses to Change during Dynamic Transtibial Prosthetic Alignment in a Clinical Setting. Prosthet. Orthot. Int. 28, 105-114 (2004)

5. Hannah, R.E., Morrison, J.B., Chapman, A.E.: Prosthesis Alignment: Effect on Gait of Persons with Below-knee Amputations. Arch. Phys. Med. Rehabil. 65, 159-162 (1984)

6. Hansen, A.H., Childress, D.S.: Effects of Shoe Heel Height on Biologic Rollover Characteristics during Walking. J. Rehab. Res. Dev. 4, 547-553 (2004)

7. Blumentritt, S., Schmalz, T., Jarasch, R., Schneider, M.: Effects of sagittal plane prosthetic alignment on standing trans-tibial amputee knee loads. Prosthet. Orthot. Int. 23, 231-238 (1999) 